\title{
A Survey on Various Clustering Algorithms in WSN for Optimal Energy Utilisation
}

\author{
Madhuri N. Khuspare, Dr. Awani S. Khobragade \\ Department of Electronics Engineering, Priyadarshini College of Engineering, Nagpur, Maharashtra, India
}

Article Info

Volume 8, Issue 3

Page Number : 712-720

\section{Publication Issue}

May-June-2021

\section{Article History}

Accepted : 10 June 2021

Published : 17 June 2021

\section{ABSTRACT}

Wireless sensor networks comprise of an expansive number of distributed sensor gadgets, which are associated and composed through multi-hop steering. Because of the presence of related data and excess in measuring data, data messages can be joined and converged by performing data aggregation work in the steering procedure. To diminish energy utilization is a noteworthy enhancement target of data aggregation approaches, which can be accomplished by diminishing the mandatory correspondence load of steering. To improvise the network lifetime as much as possible in Wireless Sensor Networks (WSNs) the ways for data move are picked in a way that the aggregate energy used along the way is limited. To help high adaptability and better data aggregation, sensor nodes are routinely collected into disjoint, noncovering subsets called clusters. Clusters make various leveled WSNs which consolidate proficient use of constrained assets of sensor nodes and in this manner broadens network lifetime. The objective of this paper is to demonstrate a forefront survey on clustering calculations announced in the writing of WSNs. This paper presents different energy effective clustering calculations in WSNs. From the hypothetical level, an energy show is proposed to approve the advantages of data aggregation on energy utilization. The key parameters which may affect the aggregation execution are additionally examined.

Keywords : Clustering, Load balancing, Fault Tolerance, Latency, Data Aggregation, LEACH, PEGASIS, TEEN, HEED

\section{INTRODUCTION}

A wireless sensor network comprises of sensor nodes conveyed over a topographical territory for observing physical marvels like temperature, moistness, vibrations, seismic occasions et cetera. Ordinarily, a sensor hub is a moment gadget that comprises of three parts, for example, a detecting subsystem for data achievement from the physical encompassing condition, a preparing subsystem for neighborhood data handling and capacity and a wireless correspondence subsystem for data transmission. 
Moreover, an energy source supplies the energy required by the gadget to play out the arranged errand. Energy utilization is one of the greatest requirements of the wireless sensor hub and this restriction joined with an ordinary sending of substantial number of nodes has added many difficulties to the plan and management of wireless sensor networks.

Clustering has turned out to be a proficient technique that expands the network life time by dropping the energy use and gives the fundamental adaptability. To accomplish high adaptability and expanded energy proficiency and to improve the network life time the scientists have profoundly embraced the plan of shaping clusters i.e. gathering the sensor nodes in extensive scale wireless sensor network situations.

Fundamentally, a clustering plan decides an arrangement of nodes that can give a spine to interface the network to the base station. The sort of nodes talked about here is called cluster heads and the rest of the nodes of the network are alluded to as part nodes.

In this clustering plan the part nodes sporadically transmit there data to the heads of the clusters they have a place and it turns into the obligation of the cluster head to total this data and transmit it to the base station. This transmission can either be immediate or by means of other cluster heads. This plan in the long run makes two level structures where larger amount constitutes of the cluster head nodes and the part nodes turn into a piece of lower level chain of importance accordingly diminishing the quantity of handed-off bundles. A cluster head hub has an extra load as it must acknowledge messages from its cluster individuals, total them, and communicate the accumulated message to the following bounce towards the sink and transfer the amassed messages began by other cluster head nodes. Re-clustering the network is regularly fundamental keeping in mind the end goal to accomplish the load balancing.

Perfect usage of clustering is dependably energy proficient if the cluster heads are fittingly situated in this way the position of cluster head turns into a key criteria in clustering for accomplishing energy productivity. In clustering plan the cluster head nodes are chosen from one of the conveyed sensors in the network where this network is homogenous in nature [1], [2].

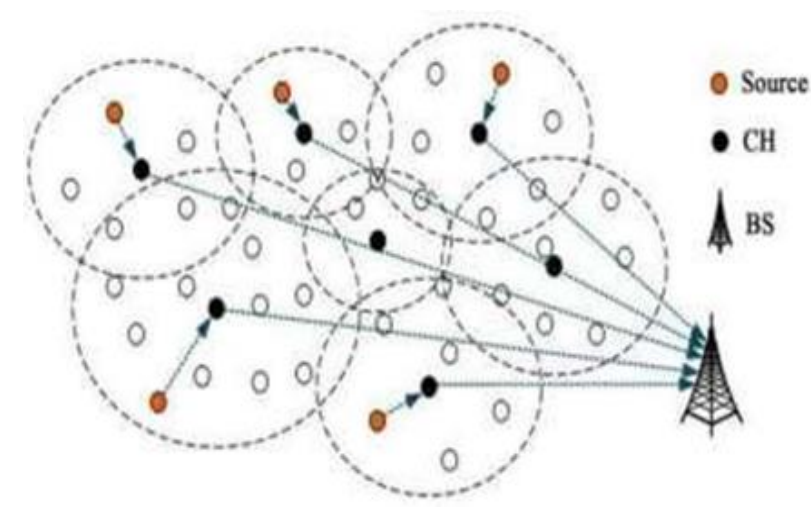

Figure 1: General Sensor Network Architecture

Correspondence region and separation from base station are real worries that need to consider while executing clustering in wireless sensor network. Another key part of clustering is the correspondence between the cluster head and the base station, if this is not immediate than Multihop steering is required which creates the significance of between cluster head network. And furthermore the cluster head ought not to be depleted superfluously which may some way or another prompt pointless loss of energy of cluster head nodes [3], [4].

\section{LITERATURE REVIEW}

Among the issues in WSN the use of energy is a champion among the most basic issues. Concerning energy proficiency, Hierarchical directing conventions are seen to be the best. By the usage of a clustering procedure they limit the use of energy 
phenomenally in social occasion and spreading the data. Various levelled directing conventions lessen the energy use by separating nodes into clusters. In each cluster, a hub having the colossal handling power is picked as a cluster head, which adds up to the data sent by the fuelled sensor nodes. In this area cluster based steering conventions for remote sensor frameworks are analysed.

In [10] Authors displayed the LEACH (Low Energy Adaptive Clustering Hierarchy) convention for WSNs of cluster-based design, which is a by and large known and exquisite clustering calculation, by choosing the $\mathrm{CHs}$ in rounds. $\mathrm{LEACH}$ is a standard energy proficient adaptive clustering calculation that structures nodes bunches in view of the flag quality and uses these nearby cluster heads as switches to the SINK. Since data trade to the base station eats up more energy, all the sensor nodes inside a cluster alternate with the transmission by turning the gathering heads. This prompts adjusted energy use of all nodes and from now on a more expanded lifetime of the framework. A predefined esteem, $\mathrm{P}$ (the coveted level of cluster heads in the network), is set before starting this calculation. LEACH works in a few rounds where each round has two phases, the setup arrange and the unfaltering stage. In the midst of the setup organize, every hub picks whether to wind up a cluster head or not. Every hub picks an arbitrary number $p$ in the vicinity of 0 and 1 , which is simply the probability to choose as a cluster head. In the event that the likelihood $\mathrm{p}$ is not as much as a limit $\mathrm{T}(\mathrm{n})$ for hub $\mathrm{n}$, hub $\mathrm{n}$ will turn into a cluster head for the current round $r$. This $\mathrm{T}(\mathrm{n})$ is ascertained by utilizing the Equation as follows:

$$
T(n)= \begin{cases}\frac{p}{1-p *\left(\operatorname{rmod} \frac{1}{p}\right)} & \text { if } n \in G \\ 0 & \text { Otherwise }\end{cases}
$$

Amid the consistent stage, the sensor nodes can begin detecting and transmitting data to the cluster heads. The cluster heads additionally total data from the sensor nodes in their cluster and sends data to the base station. After a particular time allotment spent on the enduring stage, the network goes into another round of picking the cluster heads. The length of the relentless stage is longer than the traverse of the setup stage with a particular ultimate objective to limit the overhead. LEACH gives an enhanced conduct to correspondence in WSNs considering self association methods. Versatility is additionally bolstered by LEACH, however new nodes must be synchronized to the current round. Hub disappointments may incite less cluster heads to be picked than looked for in light of the way that the predefined $\mathrm{P}$ is a level of the total number of sensor nodes.

Considering a solitary round of LEACH, a stochastic cluster- head decision won't thusly incite slightest energy usage in the midst of the consistent stage for data exchange of a given game plan of sensor nodes. For example, a segment of the cluster heads can be arranged near the edges of the network or some contiguous nodes can move toward becoming cluster heads. In these cases, some sensor nodes are further a long way from a cluster head. Regardless, considering at least two alters, a decision of positive cluster heads at the current round can realize a troublesome cluster- heads assurance in the later round. Concerning energy use, a deterministic cluster-head decision calculation can play out a stochastic calculation. The difference in the edge condition by the rest of the energy may raise another issue. Since the rest of the nodes have a low energy level after various rounds, the cluster - head limit will end up being too low. Some cluster heads won't have enough energy to transmit data to the base station. The network can't work honourably regardless of the way that there are still nodes available with enough energy to play out this errand. The edge condition can be refreshed further by consolidating a factor that raises the limit for any hub that has not been a cluster head for a specific number of rounds. The likelihood 
of this hub transforming into a cluster head extends because of the higher edge.

In [11] creators proposed Power-proficient gathering in sensor data frameworks (PEGASIS), which is a change over the LEACH. It is chain based convention, in which nodes need to talk with their closest neighbors and exchange in talking with BS. Each hub in the framework uses flag quality to discover the closest Neighbor. The chain in PEGASIS includes nodes closest to each other that shape a route to the BS. The gathered sort of the data will be sent to the BS by any hub in the chain and the nodes in the bind will interchange sending to the BS. This lessens the power required to transmit data per round in light of the fact that the power draining is spread reliably finished all nodes. In any case, the presumptions in PEGASIS may not for the most part be reasonable.

- $\quad$ PEGASIS expect that each sensor hub can talk with the BS straightforwardly. In functional cases, sensor nodes utilize multi-jump correspondence to accomplish the BS.

- It considers that all nodes keep up an entire database about the area of each other hub in the framework; however the technique by which the hub area are gotten is not portrayed.

- It considers that all sensor nodes have a similar level of energy and are probably going to pass on in the meantime.

Regardless of the way that by and large sensors will be settled or stationary as accepted in PEGASIS, a couple of sensors may be allowed to move and along these lines impact the convention capacities.

Here in [12] creators proposed a progressive clustering based convention delivered for responsive frameworks in which nodes react in a flash to sudden and extraordinary changes in condition known as TEEN. Cluster arrangement and data exchange are done as in the LEACH limit esteems close by various qualities - Hard Threshold (HT) and Soft Threshold
(ST). These qualities and in addition the earth are detected by the nodes ceaselessly. Exactly when the hub finds that the recognized characteristic has accomplished HT, the hub switches on its transmitter and sends the detected data.

The detected esteem is secured in an inward factor SV in the hub. In the present cluster time frame, the hub will next transmit data exactly when the present estimation of the detected trait is higher than HT and the present estimation of the detected characteristic differs from SV by an aggregate comparable to or higher than the ST. The use of HT and ST will diminish the quantity of transmissions in the network and therefore it decreases the general energy dissemination in the network. This arrangement is suited for time basic data detecting applications.

This Paper [13] Adaptive Periodic ThresholdSensitive Energy Efficient Sensor Network plot (APTEEN) is a growth to TEEN and goes for both sending incidental intermittent and react to basic circumstances. On the other hand, APTEEN merges the part of proactive and responsive frameworks and transmits data in adjustable time breaks while in any case it responds to sudden changes in characteristic esteems. APTEEN relies upon an inquiry system which licenses three sorts of request: recorded ontime and consistent which can be used as a piece of a half breed framework. The $\mathrm{CH}$ decision framework relies upon the method used as a piece of LEACH

C. In APTEEN, CHs broadcast the four parameters: Attributes, Thresholds, Schedule and Count Time.

All nodes in APTEEN sense nature reliably, yet the data transmission happens exactly when distinguished data quality is at or more conspicuous than HT. For a hub, if a data transmission does not happen in day and age proportional to the number time, it must detect and transmit the data yet again. In APTEEN, each $\mathrm{CH}$ aggregates the data from the part nodes inside its cluster and transmits the amassed data to the BS. The convention acknowledges that the data got from part 
nodes are sufficiently corresponded; thusly it diminishes a ton of abundance of the data to be sent to the BS. Plus, a balanced TDMA design realizes the half and half framework by assigning transmission space to all nodes in a cluster. In addition, APTEEN offers a lot of adaptability by allowing the clients to set the CT between time and the edge esteems for energy usage can be controlled by changing the CT and what's more the farthest point esteems.

Here in [14] creators proposed a distributed, randomized clustering calculation for WSNs. This technique is apportioned into two phases specifically single-level clustering and multi-level clustering. In the single-level clustering, each sensor hub pronounces itself as a $\mathrm{CH}$ with likelihood $\mathrm{p}$ to the neighboring hub inside its correspondence run. These $\mathrm{CHs}$ are named as the volunteer $\mathrm{CHs}$. All nodes that are inside $\mathrm{k}$ jumps scope of a $\mathrm{CH}$ get this declaration either by coordinate correspondence or by sending. Constrained $\mathrm{CHs}$ are not nodes that are neither $\mathrm{CH}$ nor have a place with a cluster. In case the declaration does not accomplish a hub inside a pre-set time interim $t$ that is figured in light of the term for a bundle to achieve a hub that is $\mathrm{k}$ jumps away, the hub will transform into a constrained $\mathrm{CH}$ expecting that it is not inside $\mathrm{k}$ bounces of all volunteer CHs. The second stage, called multi-level clustering constructs $\mathrm{h}$ levels of cluster progression. The calculation ensures $\mathrm{h}$ - bounce network among $\mathrm{CHs}$ and the base station. The $\mathrm{CHs}$ closest to the base station have burden since they go about as transfers for various CHs.

Paper [15] is an another noticeable energy-capable hub clustering calculation is the Hybrid, Energy Efficient and Distributed (HEED) clustering approach for impromptu sensor networks. HEED made by [21] is a distributed clustering convention which was proposed with four fundamental goals as follows:

- Expanding network life expectancy by dispersing energy usage,
- Finishing the clustering system inside a consistent number of cycles,

- Diminishing control overhead (to be direct in the quantity of nodes),

- $\quad$ Producing great - distributed cluster heads and reduced clusters.

HEED occasionally picks cluster heads in view of a cross breed of two clustering parameters: The fundamental parameter is the remaining energy of each sensor hub and the optional parameter is the intra-cluster correspondence cost as a segment of Neighbor region or cluster thickness. The essential parameter is used to probabilistically pick an underlying arrangement of cluster heads while the auxiliary parameter is utilized for breaking ties.

The gathering methodology at each sensor hub requires a couple of rounds. Each round is adequately long to get messages from any Neighbor inside the cluster extend. As in LEACH, a hidden rate of cluster heads in the framework, Cprob, is predefined. The parameter Cprob is quite recently used to compel the basic cluster head declarations and has no immediate impact on the last cluster structure. In HEED, every sensor hub sets the likelihood CHprob of turning into a cluster head as follows Where Eresidual is the evaluated current lingering energy in this sensor hub and Emax is the most extreme energy (relating to a completely charged battery), which is ordinarily indistinguishable for homogeneous sensor nodes. The CHprob esteem must be more noteworthy than a base edge $\mathrm{p}$ min. A cluster head is either a provisional cluster - head, if its CHprob is $<1$, or a last cluster head, if its CHprob has achieved 1.

In the midst of each round of HEED, every sensor hub that never got notice by the $\mathrm{CH}$ picks the same hub to end up plainly a $\mathrm{CH}$ with the same CHprob. The as of late picked $\mathrm{CH}$ are added to the present game plan of cluster heads. In the event that a sensor hub is wound up a gathering $\mathrm{CH}$, it sends a declaration message as a conditional $\mathrm{CH}$ or a last $\mathrm{CH}$. 
A node tuning in to the $\mathrm{CH}$ list picks the cluster head with the most negligible cost from this course of action of $\mathrm{CH}$. Each hub at that point duplicates its $\mathrm{CHprob}$ and goes to the subsequent stage. On the off chance that a hub executes the HEED except picking itself to end up plainly a $\mathrm{CH}$ or joining a group, it pronounces itself as a last $\mathrm{CH}$. A provisional $\mathrm{CH}$ hub can turn into a general hub at an upcoming cycle in the event that it gets notification from a less energy consuming $\mathrm{CH}$. Remember that hub may be picked as a $\mathrm{CH}$ at upcoming clustering interims on the off chance that it has high energy with less cost. As the WSNs is believed to be a static network, where hub doesn't pass on all of a sudden, the node set near every hub does not alter sometimes.

Now HEED should not be dynamically changing hub. The circulation of resource use of HEED increments the life of the considerable number of hubs in the network. Nodes likewise consequently refresh their neighbour sets in multi-jump network sometimes by sending and accepting messages. The HEED system improvises the architectures lifetime over LEACH since it heedlessly picks $\mathrm{CH}$ (and henceforth the sizes of cluster), that leads to early death of a couple of hubs. The last $\mathrm{CH}$ picked here are especially coursed over the system and the correspondence investment is limited.

In [16] creators proposed first unequal clustering model, called Unequal Clustering Size (UCS) to change energy use. The sensor field is isolated into two concentric circles called layers and each layer has some number of clusters of same size. The size and states of the clusters of two layers are unmistakable. The convention accept that the BS is arranged in the inside purpose of the framework and $\mathrm{CHs}$ regions are resolved "priori" which are arranged symmetrically in concentric circles around the BS. To limit the energy use inside the cluster, each $\mathrm{CH}$ should be set at the middle purpose of the cluster. $\mathrm{CHs}$ are deterministically planted in the framework and are believed to be super nodes which are significantly more expensive than part nodes. The scope of the clusters can be moved by contrasting the first of the essential layer around the BS, so the quantity of nodes in a particular cluster likewise changed. Each $\mathrm{CH}$ transmits data to BS by picking the closest $\mathrm{CH}$ toward BS.

The UCS has two inclinations stood out from LEACH. In any case, the UCS can keep up uniform energy usage among CHs. This can be refined by varying the quantity of nodes in each cluster concerning the typical correspondence load. Likewise, convention influences two layered network to model and twojump between cluster specialized strategy, this result in a shorter normal transmission remove differentiated and LEACH, along these lines effectively reduces the entire energy use.

In [17] creators proposed the flow-balanced routing (FBR) convention for multi-jump clustered WSNs. The convention attempts to fulfill both power productivity and scope defending. The convention includes four phases: network clustering, multibounce spine development, flow adjusted transmission and rerouting. The few nodes are assembled into one cluster on the premise of covering degrees of sensors. In spine improvement organize; a novel multi-level spine is assembled using the $\mathrm{CHs}$ and the BS. The flow-adjusted directing allocates the exchanged data over numerous ways from the sensors to the BS in order to try and out the power use of sensors. Exactly when the $\mathrm{CH}$ missed the mark on energy, the $\mathrm{CH}$ drops out from the spine and in such places the network topology is reconfigured in rerouting stage. The two estimations called the network lifetime and the scope lifetime are considered to evaluate the accomplishment of FBR convention. The reproduction comes about show that FBR yields both longer lifetime and better scope shielding. 
Here in [18] creators proposed CBRP conspire in which the framework is clustered by using a couple of parameters and after that building up a spreading over tree for sending amassed data to the base station. The operation of CBRP is parcelled into two phases, for instance, Cluster head decision organize and steering tree time arrange. In the $\mathrm{CH}$ decision arrange the $\mathrm{CH}$ determination relies upon the Cluster Head Selection Value (CHSV), the greatest CHSV esteem hub will transform into the cluster head. In steering tree time arrange each cluster head will pick their parent sensor hub in light of the Parent Selection Value (PSV). Next, the steering tree is constructed and the transmission happens. CBRP considers the separation and leftover energy of nodes and picks perfect $\mathrm{CHs}$ that can save more energy in nodes. Trial comes about show that CBRP equalities the energy use among $\mathrm{CHs}$ and in this way more energy is saved in the framework.

In [19] creators proposed an Energy Efficient Clustering Scheme (EECS) for the periodical data gathering applications. In EECS, the framework is allotted into various clusters and uses single-bounce correspondences between the $\mathrm{CH}$ and the BS. In EECS, $\mathrm{CH}$ candidates seek the ability to hoist $\mathrm{CH}$ for a given round. Each $\mathrm{CH}$ hopefuls demonstrate their left energy to neighbouring candidates. If a given hub does not find a hub with more extra energy, it transforms into a CH. EECS widens LEACH by powerful measuring of gatherings in perspective of cluster separate from the BS. The intra-cluster correspondence cost is lessened by picking the closest $\mathrm{CH}$.

In [20] Power-Efficient and Adaptive Clustering Hierarchy $(\mathrm{PEACH})$ tradition is proposed for WSNs to widen framework lifetime by decreasing the energy usage. The nodes in the framework can see the source and goal of the data parcels by catching characteristics of wireless correspondence. In PEACH, the clusters are surrounded without additional transmission overhead, for instance, see, assertion, joining and booking messages. PEACH is probabilistic coordinating

calculation and give an adaptable multi-level clustering. PEACH is amazingly capable and versatile under unexpected conditions in comparison to the present clustering conventions.

PEACH may be suitable to both mindful and unconscious WSNs regarding area. In particular applications, the area data of the hub is not known. In such applications, area uninformed PEACH tradition can be used. The area mindful PEACH works when the restriction instrument, for instance, a GPS- like hardware is available on sensor nodes.

\section{CONCLUSION}

Specialists have been lured towards wireless sensor networks in later past both in scholarly and mechanical areas. The plan of compelling, strong, and versatile steering conventions for WSNs is a testing undertaking. Then again, clustering directing calculations, for the most part, can well match the imperatives and the difficulties of WSNs. Accordingly; it is plainly observed so far that, critical endeavors have been made in tending to the systems to outline compelling and effective clustering directing conventions for WSNs in the previous couple of years. This paper have reviewed the condition of-specialty of various clustering calculations in wireless sensor networks alongside LEACH and other critical conventions detailed in the writing of WSNs till today. Each exertion has been made to give finish and exact cutting edge review on energy productive clustering calculations as relevant to WSNs.

\section{REFERENCES}

[1]. W.B. Heinzelman, A.P. Chandrakasan and H. Balakrishnan, "Application specific protocol architecture for wireless microsensor 
networks", IEEE Transactions on Wireless Communications, vol.1, no.4, Oct 2002, pp.660670.

[2]. S. Lindsey and C.S. Raghavendra, "PEGASIS:Power efficient gathering in sensor information system", in Proc. of IEEE Aerospace conference, vol.3, March 2002, pp.1125-1130.

[3]. S. Banerjee and S. Khuller, "A clustering scheme for hierarchical control in multihop wireless networks", in Proc. of 20th Annual Joint Conference of the IEEE Computer \& Communications Societies (INFOCOM'01), vol.2, April 2001, pp.1028-1037.

[4]. S. Banbyopadhyay and E.J. Coyle, "An energy efficient hierarchical clustering algorithm for wireless sensor networks", Twenty-Second Annual Joint Conference of the IEEE Computer and Communications IEEE Societies (INFOCOM 2003), vol.3, April 2003, pp.17131723.

[5]. S.K. Singh, M.P. Singh and D.K. Singh, "Routing protocols in Wireless Sensor Networks - a Survey" International journal of computer science \& engineering survey (IJCSES) vol.1, no.2, Nov 2010, pp.63-83.

[6]. R. Rajagopalan and P.K. Varshney, "Data Aggregation Techniques in Sensor Networks: A Survey", IEEE Communications Surveys and Tutorials, vol. 8, no. 4, Oct 2006, pp. 48-63.

[7]. J. Yuea, W. Zhang, W. Xiao, D. Tang and J. Tang, "Energy Efficient and balanced clusterbased data aggregation algorithm for wireless Sensor Networks", 2012 International Workshop on Information and Electronics Engineering, Procedia Engineering, vol. 29, 2012, pp.2009- 2015.

[8]. J. Li and P. Mohapatra, "Analytical modeling and mitigation techniques for energy hole problem in sensor networks", Pervasive Mobile Computing, vol. 3, no. 3, June 2007, pp. 233254.
[9]. S.H. Lee, S. Lee, H. Song and H.S. Lee, "Gradual Cluster Head Election for High Network Connectivity in Large- Scale Sensor Networks", Proceedings of 13th International Conference on Advanced Communication Technology (ICACT), Phoenix Park, Korea, Feb 2011 pp. 168-172.

[10]. W. Heinzelman, A. Chandrakasan and H. Balakrishnan, "Energy- efficient Communication Protocol for Wireless Microsensor Networks", Proceedings of the 33rd Annual Hawaii International Conference on System Sciences, 2000.

[11]. S. Lindsey and C.S. Raghavendra, "PEGASIS:Power efficient gathering in sensor information system", in Proc. of IEEE Aerospace conference, vol.3, March 2002, pp.1125-1130.

[12]. A. Manjeshwar and D.P. Agrawal, "TEEN: A Routing Protocol for Enhanced Efficiency in Wireless Sensor Networks", Proceedings of the15th International Parallel \& Distributed Processing Symposium, IEEE Computer Society, April 2000, pp. 2009-2015.

[13]. A. Manjeshwar and D. P. Agarwal, "APTEEN: A hybrid protocol for efficient routing and comprehensive information retrieval in wireless sensor networks," in Proceedings of the 2nd International Workshop on Parallel and Distributed Computing Issues in Wireless Networks and Mobile computing, FL, USA, April 2002, pp.195-202.

[14]. S. Banbyopadhyay and E.J. Coyle, "An energy efficient hierarchical clustering algorithm for wireless sensor networks", Twenty-Second Annual Joint Conference of the IEEE Computer and Communications IEEE Societies (INFOCOM 2003), vol.3, April 2003, pp.17131723.

[15]. O. Younis and S. Fahmy, "HEED: A Hybrid, Energy- Efficient, Distributed Clustering Approach for Ad Hoc Sensor Networks", IEEE 
Transactions on Mobile Computing, vol.3, no.

4, Oct 2004, pp.366-379.

[16]. S. Soro and W.B. Heinzelman, "Prolonging the lifetime of wireless sensor networks via unequal clustering," in Proceedings of 19th IEEE International Parallel and Distributed Processing Symposium, April 2005.

[17]. Y. Tao, Y. Zhang and Y. Ji, "Flow-balanced routing for multi-hop clustered wireless sensor networks," Ad Hoc Networks, vol.11, no.1, January 2013, pp. 541-554.

[18]. B. Zarei, M. Zeynali and V.M. Nezhad, "Novel Cluster Based Routing Protocol in Wireless Sensor Networks", IJCSI International Journal of Computer Science, vol.7, no.4, 2010.

[19]. M. Ye, C. Li, G. Chen and J. Wu, "An energy efficient clustering scheme in wireless sensor networks," Ad Hoc and Sensor Wireless Networks, vol. 3, April 2006, pp.99- 119.

[20]. Y. Sangho, H. Junyoung, C. Yookun and J. Hong, "PEACH: Power- efficient and adaptive clustering hierarchy protocol for wireless sensor networks," Computer Communications, vol. 30, no.14-15, October 2007, pp. 2842-2852.

[21]. A.U. Khattak, G.A. Shah and M. Ahsan, "Twotier cluster based routing protocol for wireless sensor networks," in Proceedings of IEEE/IFIP 8th International Conference on Embedded and Ubiquitous Computing (EUC), Hong Kong, December 2010, pp. 410-415.

[22]. N. Gautam and J.Y. Pyun, "Distance aware intelligent clustering protocol for wireless sensor networks," IEEE Journal of Communications and Networks, vol.12, no.2, April 2010, pp. 122-129.

\section{Cite this article as :}

Madhuri N. Khuspare, Dr. Awani S. Khobragade, "A Survey on Various Clustering Algorithms in WSN for Optimal Energy Utilisation", International Journal of Scientific Research in Science and Technology (IJSRST), Online ISSN : 2395-602X, Print ISSN : 23956011, Volume 8 Issue 3, pp. 712-720, May-June 2021. Available

doi : https://doi.org/10.32628/IJSRST2183153

Journal URL : https://ijsrst.com/IJSRST2183153 